\title{
Periodic Patterns of Signed Shifts
}

\author{
Kassie Archer and Sergi Elizalde \\ Department of Mathematics, Dartmouth College, Hanover, NH, USA
}

\begin{abstract}
The periodic patterns of a map are the permutations realized by the relative order of the points in its periodic orbits. We give a combinatorial description of the periodic patterns of an arbitrary signed shift, in terms of the structure of the descent set of a certain transformation of the pattern. Signed shifts are an important family of one-dimensional dynamical systems. For particular types of signed shifts, namely shift maps, reverse shift maps, and the tent map, we give exact enumeration formulas for their periodic patterns. As a byproduct of our work, we recover some results of Gessel and Reutenauer and obtain new results on the enumeration of pattern-avoiding cycles.

Résumé. Les motifs périodiques d'une fonction sont les permutations réalisées par l'ordre relatif des points dans ses orbites périodiques. Nous donnons une description combinatoire des motifs périodiques d'un shift signé arbitraire, en termes de la structure de l'ensemble des descentes d'une certaine transformation du motif. Les shifts signés sont une familie importante de systèmes dynamiques unidimensionnels. Pour des types particuliers de shifts signés, comme les fonctions de shift, les fonctions de shift inversées, et la fonction de tente, nous donnons des formules exactes pour l'énumération de leurs motifs périodiques. Comme sous-produit de notre travail, nous retrouvons des résultats de Gessel et Reutenauer et obtenons de nouveaux résultats sur l'énumération de cycles qui évitent certain motifs.
\end{abstract}

Keywords: periodic pattern; signed shift; cyclic permutation; descent; pattern avoidance.

\section{Introduction}

\subsection{Background and motivation}

Permutations realized by the orbits of a map on a one-dimensional interval have received a significant amount of attention in the last five years [2]. These are the permutations given by the relative order of the elements of the sequence obtained by successively iterating the map, starting from any point in the interval. One the one hand, understanding these permutations provides a powerful tool to distinguish random from deterministic time series, based on the remarkable fact [6] that every piecewise monotone map has forbidden patterns, i.e., permutations that are not realized by any orbit. Permutation-based tests for this purpose have been developed in [4]. On the other hand, the set of permutations realized by a map (also called allowed patterns) has a rich combinatorial structure. The answer to certain enumerative questions, often involving pattern-avoiding permutations, provides information about the associated dynamical systems. For example, determining the asymptotic growth of the number of allowed patterns of a map reveals its so-called topological entropy, an important measure of the complexity of the system.

\footnotetext{
$\dagger$ Partially supported by NSF grant DMS-1001046.

1365-8050 @ 2013 Discrete Mathematics and Theoretical Computer Science (DMTCS), Nancy, France
} 
The dynamical systems most commonly studied from the perspective of forbidden patterns are shifts, and more generally signed shifts [1], a large family of maps that includes the tent map (which is equivalent to the logistic map). As we will see, signed shifts have a simple discrete structure which makes them amenable to a combinatorial approach, yet they include many important chaotic dynamical systems.

Permutations realized by shifts were first considered in [3], and later characterized and enumerated in [8]. More recently, permutations realized by the more general $\beta$-shifts have been studied in [9]. For the logistic map, some properties of their set of forbidden patterns were given in [10].

If instead of considering an arbitrary initial point in the domain of the map we restrict our attention to periodic points, the permutations realized by the relative order of the entries in the corresponding orbits (up until the first repetition) are called periodic patterns. In the case of continuous maps, Sharkovskii's theorem [13] gives a beautiful characterization of the possible periods of these orbits. More refined results that consider which periodic patterns are forced by others are known for continuous maps [7, 12]. However, little is known when the maps are not continuous, as is the case for shifts and, more generally, signed shifts.

The subject of study of this paper are periodic patterns of signed shifts. Our main result is a characterization of the periodic patterns of an (almost) arbitrary signed shift, given in Theorem 2.1. For some particular cases of signed shifts we obtain exact enumeration formulas: the number of periodic patterns of the tent map is given in Theorem 3.2, and the number of periodic patterns of the (unsigned) shift map is given in Theorem 3.5 For the reverse shift, which is not covered in our main theorem, the number of periodic patterns is studied in Sections 3.3 and 3.4 .

An interesting consequence of our study of periodic patterns is that we obtain new results regarding the enumeration of cyclic permutations that avoid certain patterns. These are described in Section 4

\subsection{Periodic patterns}

Given a linearly ordered set $X$ and a map $f: X \rightarrow X$, consider the sequence $\left\{f^{i}(x)\right\}_{i \geq 0}$ obtained by iterating the function starting at a point $x \in X$. If there are no repetitions among the first $n$ elements of this sequence, called the orbit of $x$, then we define the pattern of length $n$ of $f$ at $x$ to be

$$
\operatorname{Pat}(x, f, n)=\operatorname{st}\left(x, f(x), f^{2}(x), \ldots, f^{n-1}(x)\right),
$$

where st is the operation that outputs the permutation of $[n]=\{1,2, \ldots, n\}$ whose entries are in the same relative order as $n$ entries in the input. For example, st $(3.3,3.7,9,6,0.2)=23541$. If $f^{i}(x)=f^{j}(x)$ for some $0 \leq i<j<n$, then $\operatorname{Pat}(x, f, n)$ is not defined. The set of allowed patterns of $f$ is $\mathcal{A}(f)=$ $\{\operatorname{Pat}(x, f, n): n \geq 0, x \in X\}$.

We say that $x \in X$ is an n-periodic point of $f$ if $f^{n}(x)=x$ but $f^{i}(x) \neq x$ for $1 \leq i<n$. In this case, the permutation $\operatorname{Pat}(x, f, n)$ is denoted $\operatorname{PP}(x, f)$, and called the periodic pattern of $f$ at $x$. Note that if $x$ is an $n$-periodic point, then $\operatorname{Pat}(x, f, i)$ is not defined for $i>n$. Let $\mathcal{P}(f)=\{\operatorname{PP}(x, f): x \in X\}$ be the set of periodic patterns of $f$, and let $\mathcal{P}_{n}(f)=\mathcal{P}(f) \cap \mathcal{S}_{n}$. For a permutation $\pi=\pi_{1} \pi_{2} \ldots \pi_{n} \in \mathcal{S}_{n}$, let $[\pi]=\left\{\pi_{i} \pi_{i+1} \ldots \pi_{n} \pi_{1} \ldots \pi_{i-1}: 1 \leq i \leq n\right\}$ the set of cyclic rotations of $\pi$, which we call the equivalence class of $\pi$. It is clear that if $\pi \in \mathcal{P}(f)$, then $[\pi] \subset \mathcal{P}(f)$. Indeed, if $\pi$ is the periodic pattern at a point $x$, then the other permutations in $[\pi]$ are realized at the other points in the periodic orbit of $x$. Let $\overline{\mathcal{P}}_{n}(f)=\left\{[\pi]: \pi \in \mathcal{P}_{n}(f)\right\}$ denote the set of equivalence classes of periodic patterns of $f$ of length $n$, and let $p_{n}(f)=\left|\overline{\mathcal{P}}_{n}(f)\right|=\left|\mathcal{P}_{n}(f)\right| / n$.

Given linearly ordered sets $X$ and $Y$, two maps $f: X \rightarrow X$ and $g: Y \rightarrow Y$ are said to be orderisomorphic if there is an order-preserving bijection $\phi: X \rightarrow Y$ such that $\phi \circ f=g \circ \phi$. In this 
case, $\operatorname{Pat}(x, f, n)=\operatorname{Pat}(\phi(x), g, n)$ for every $x \in X$ and $n \geq 1$. In particular, $\mathcal{A}(f)=\mathcal{A}(g)$ and $\mathcal{P}(f)=\mathcal{P}(g)$.

\subsection{Signed shifts}

Let $k \geq 2$ be fixed, and let $\mathcal{W}_{k}$ be the set of infinite words $s=s_{1} s_{2} \ldots$ over the alphabet $\{0,1, \ldots, k-1\}$. Let $<_{\text {lex }}$ denote the lexicographic order on these words. We use the notation $s_{[i, \infty)}=s_{i} s_{i+1} \ldots$, and $\bar{s}_{i}=k-1-s_{i}$. If $q$ is a finite word, $q^{m}$ denotes concatenation of $q$ with itself $m$ times, and $q^{\infty}$ is an infinite periodic word.

Fix $\sigma=\sigma_{0} \sigma_{1} \ldots \sigma_{k-1} \in\{+,-\}^{k}$. Let $T_{\sigma}^{+}=\left\{t: \sigma_{t}=+\right\}$ and $T_{\sigma}^{-}=\left\{t: \sigma_{t}=-\right\}$, and note that these sets form a partition of $\{0,1, \ldots, k-1\}$. We give two definitions of the signed shift with signature $\sigma$, and show that they are order-isomorphic to each other.

The first definition, which we denote by $\Sigma_{\sigma}^{\prime}$, is the map $\Sigma_{\sigma}^{\prime}:\left(\mathcal{W}_{k},<_{\text {lex }}\right) \rightarrow\left(\mathcal{W}_{k},<_{\text {lex }}\right)$ defined by

$$
\Sigma_{\sigma}^{\prime}\left(s_{1} s_{2} s_{3} s_{4} \ldots\right)= \begin{cases}s_{2} s_{3} s_{4} \ldots & \text { if } s_{1} \in T_{\sigma}^{+}, \\ \overline{s_{2}} \overline{s_{3}} \overline{s_{4}} \ldots & \text { if } s_{1} \in T_{\sigma}^{-} .\end{cases}
$$

It is shown in [1] that $\Sigma_{\sigma}^{\prime}$ is order-isomorphic to the piecewise linear function $M_{\sigma}:[0,1] \rightarrow[0,1]$ defined for $x \in\left[\frac{t}{k}, \frac{t+1}{k}\right)$, for each $0 \leq t \leq k-1$, as

$$
M_{\sigma}(x)= \begin{cases}k x-t & \text { if } t \in T_{\sigma}^{+}, \\ t+1-k x & \text { if } t \in T_{\sigma}^{-} .\end{cases}
$$

As a consequence, the allowed patterns and the periodic patterns of $\Sigma_{\sigma}^{\prime}$ are the same as those of $M_{\sigma}$, respectively. A few examples of the function $M_{\sigma}$ are pictured in Figure 1
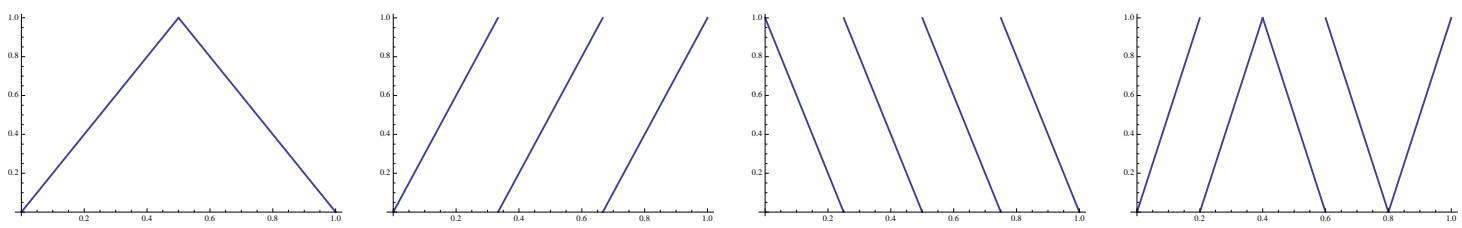

Fig. 1: The graphs of $M_{\sigma}$ for $\sigma=+-, \sigma=+++, \sigma=----$ and $\sigma=++--+$, respectively.

The second definition of the signed shift will be more convenient when studying its periodic patterns. Let $\prec_{\sigma}$ be the linear order on $\mathcal{W}_{k}$ defined by $s=s_{1} s_{2} s_{3} \ldots \prec_{\sigma} t_{1} t_{2} t_{3} \ldots=t$ if either $s_{1}<t_{1}$, $s_{1}=t_{1} \in T_{\sigma}^{+}$and $s_{2} s_{3} \ldots \prec_{\sigma} t_{2} t_{3} \ldots$, or $s_{1}=t_{1} \in T_{\sigma}^{-}$and $t_{2} t_{3} \ldots \prec_{\sigma} s_{2} s_{3} \ldots$. Equivalently, $s \prec_{\sigma} t$ if, letting $j \geq 1$ be the smallest such that $s_{j} \neq t_{j}$, either $c:=\left|\left\{1 \leq i<j: s_{i} \in T_{\sigma}^{-}\right\}\right|$is even and $s_{j}<t_{j}$, or $c$ is odd and $s_{j}>t_{j}$. The signed shift is the map $\Sigma_{\sigma}:\left(\mathcal{W}_{k}, \prec_{\sigma}\right) \rightarrow\left(\mathcal{W}_{k}, \prec_{\sigma}\right)$ defined simply by $\Sigma_{\sigma}\left(s_{1} s_{2} s_{3} s_{4} \ldots\right)=s_{2} s_{3} s_{4} \ldots$.

To show that the two definitions of the signed shift as $\Sigma_{\sigma}$ and $\Sigma_{\sigma}^{\prime}$ are order-isomorphic, consider the order-preserving bijection $\psi_{\sigma}:\left(\mathcal{W}_{k}, \prec_{\sigma}\right) \rightarrow\left(\mathcal{W}_{k},<_{\text {lex }}\right)$ that maps a word $s=s_{1} s_{2} s_{3} \ldots$ to the word $a=a_{1} a_{2} a_{3} \ldots$ where

$$
a_{i}= \begin{cases}s_{i} & \text { if }\left|\left\{j<i: s_{j} \in T_{\sigma}^{-}\right\}\right| \text {is even, } \\ \overline{s_{i}} & \text { if }\left|\left\{j<i: s_{j} \in T_{\sigma}^{-}\right\}\right| \text {is odd. }\end{cases}
$$


It is easy to check that $\psi_{\sigma} \circ \Sigma_{\sigma}=\Sigma_{\sigma}^{\prime} \circ \psi_{\sigma}$, and so $\mathcal{P}\left(\Sigma_{\sigma}\right)=\mathcal{P}\left(\Sigma_{\sigma}^{\prime}\right)$. From now on we use the second definition $\Sigma_{\sigma}$ only. The $n$-periodic points of $\Sigma_{\sigma}$ are the words of the form $s=\left(s_{1} s_{2} s_{3} \ldots s_{n}\right)^{\infty}$ where $s_{1} s_{2} \ldots s_{n}$ is a primitive word (sometimes called aperiodic word), that is, not a concatenation of copies of a strictly shorter word. Counting these words up to cyclic rotation, we obtain the following result, where $\mu$ denotes the Möbius function.

Lemma 1.1 If $\sigma \in\{+,-\}^{k}$, where $k \geq 2$, the number of periodic orbits of size $n$ of $\Sigma_{\sigma}$ is

$$
L_{k}(n)=\frac{1}{n} \sum_{d \mid n} \mu(d) k^{\frac{n}{d}}
$$

For example, if $\sigma=+--$, then $s=(00110221)^{\infty}$ is an 8-periodic point of $\Sigma_{\sigma}$, and $\operatorname{PP}\left(s, \Sigma_{\sigma}\right)=$ 12453786 , so $12453786 \in \mathcal{P}\left(\Sigma_{+--}\right)$. One of our main goals is to characterize the sets $\mathcal{P}\left(\Sigma_{\sigma}\right)$.

If $\sigma=+{ }^{k}$, then $\prec_{\sigma}$ is the lexicographic order $<_{\text {lex }}$, and $\Sigma_{\sigma}$ is called the $k$-shift. When $\sigma=-{ }^{k}$, the map $\Sigma_{\sigma}$ is called the reverse $k$-shift. When $\sigma=+-$, the map $\Sigma_{\sigma}$ is the well-known tent map.

\subsection{Pattern avoidance}

Let $\mathcal{S}_{n}$ denote the set of permutations of $[n]$, and let $\mathcal{S}=\bigcup_{n>0} \mathcal{S}_{n}$. We write permutations in one line notation as $\pi=\pi_{1} \pi_{2} \ldots \pi_{n} \in \mathcal{S}_{n}$. We say that $\tau \in \mathcal{S}_{n}$ contains $\rho \in \mathcal{S}_{m}$ if there exist indices $i_{1}<i_{2}<\cdots<i_{m}$ such that $\operatorname{st}\left(\tau_{i_{1}} \tau_{i_{2}} \ldots \tau_{i_{m}}\right)=\rho_{1} \rho_{2} \ldots \rho_{m}$. Otherwise, we say that $\tau$ avoids $\rho$. If $\mathscr{A}$ is a set of permutations, we denote by $\mathscr{A}(\rho)$ the set of permutations in $\mathscr{A}$ avoiding $\rho$, and we define $\mathscr{A}\left(\rho^{(1)}, \rho^{(2)}, \ldots\right)$ analogously as the set of permutations avoiding all the patterns $\rho^{(1)}, \rho^{(2)}, \ldots$ We say that $\mathscr{A}$ is a (permutation) class if it is closed under pattern containment, that is, if $\tau \in \mathscr{A}$ and $\tau$ contains $\rho$, then $\rho \in \mathscr{A}$. Sets of the form $\mathcal{S}\left(\rho^{(1)}, \rho^{(2)}, \ldots\right)$ are permutation classes.

Given classes $\mathscr{A}_{0}, \mathscr{A}_{1}, \ldots, \mathscr{A}_{k-1}$, their juxtaposition, denoted $\left[\mathscr{A}_{0} \mathscr{A}_{1} \ldots \mathscr{A}_{k-1}\right]$, is the set of permutations that can be expressed as concatenations $\alpha_{0} \alpha_{1} \ldots \alpha_{k-1}$ where st $\left(\alpha_{t}\right) \in \mathscr{A}_{t}$ for all $0 \leq t<k$. For example, $[\mathcal{S}(21) \mathcal{S}(12)]$ is the set of unimodal permutations, i.e., those $\pi \in \mathcal{S}_{n}$ satisfying $\pi_{1}<\pi_{2}<\cdots<$ $\pi_{j}>\pi_{j+1}>\cdots>\pi_{n}$ for some $1 \leq j \leq n$. The juxtaposition of permutation classes is again a class, and as such, it can be characterized in terms of pattern avoidance. For example, $[\mathcal{S}(21) \mathcal{S}(12)]=\mathcal{S}(213,312)$. Atkinson [5] showed that if $\mathscr{A}_{t}$ can be characterized by avoidance of a finite set of patterns for each $t$, then the same is true for $\left[\mathscr{A}_{0} \mathscr{A}_{1} \ldots \mathscr{A}_{k-1}\right]$.

Let $\sigma=\sigma_{0} \sigma_{1} \ldots \sigma_{k-1} \in\{+,-\}^{k}$ as before. We let $\mathcal{S}^{\sigma}=\left[\mathscr{A}_{0} \mathscr{A}_{1} \ldots \mathscr{A}_{k-1}\right]$ where, for $0 \leq t<k$,

$$
\mathscr{A}_{t}= \begin{cases}\mathcal{S}(21) & \text { if } \sigma_{t}=+ \\ \mathcal{S}(12) & \text { if } \sigma_{t}=-\end{cases}
$$

Let $\mathcal{S}_{n}^{\sigma}=\mathcal{S}^{\sigma} \cap \mathcal{S}_{n}$. Figure 2 shows two permutations in $\mathcal{S}^{+--}$. Note that since the empty permutation belongs to $\mathcal{S}(21)$ and to $\mathcal{S}(12)$, it is trivial that $\mathcal{S}^{+--} \subset \mathcal{S}^{+-+-}$, for example.

We denote by $\mathcal{C}_{n}$ (respectively, $\mathcal{C}^{\sigma}, \mathcal{C}_{n}^{\sigma}$ ) the set of cyclic permutations in $\mathcal{S}_{n}$ (respectively, $\mathcal{S}^{\sigma}, \mathcal{S}_{n}^{\sigma}$ ). In Figure 2, the permutation on the right is in $\mathcal{C}^{\sigma}$. It will be useful to define the map

$$
\begin{aligned}
\theta: \mathcal{S}_{n} & \rightarrow \mathcal{C}_{n} \\
\pi & \mapsto \hat{\pi},
\end{aligned}
$$

where if $\pi=\pi_{1} \pi_{2} \ldots \pi_{n}$ in one-line notation, then $\hat{\pi}=\left(\pi_{1}, \pi_{2}, \ldots, \pi_{n}\right)$ in cycle notation, that is, $\hat{\pi}$ is the cyclic permutation that sends $\pi_{1}$ to $\pi_{2}, \pi_{2}$ to $\pi_{3}$, and so on. Writing $\hat{\pi}=\hat{\pi}_{1} \hat{\pi}_{2} \ldots \hat{\pi}_{n}$ in one-line 

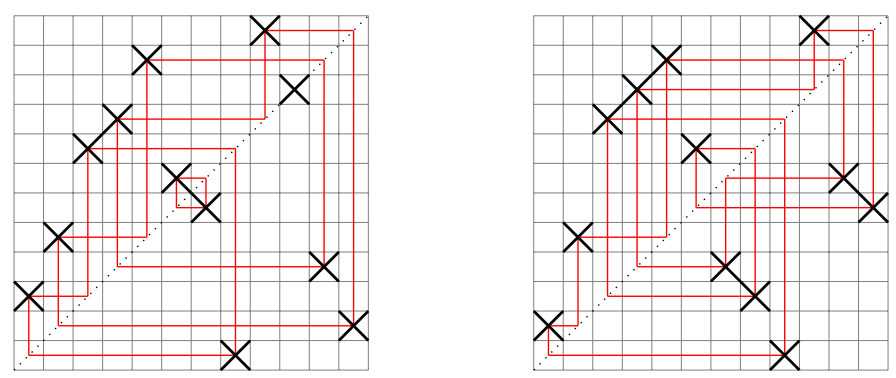

Fig. 2: Two permutations 358911761121042 and 259101184311276 in $\mathcal{S}^{+--}$and their cycle structure.

notation, we have that $\hat{\pi}_{\pi_{i}}=\pi_{i+1}$ for $1 \leq i \leq n$, with the convention that $\pi_{n+1}:=\pi_{1}$. The map $\theta$ also plays an important role in [8]. Note that if $\pi \in \mathcal{S}_{n}$, then $\theta^{-1}(\hat{\pi})=[\pi]$, the set of cyclic rotations of $\pi$.

\section{Description of periodic patterns of the signed shift}

The main theorem of this paper is the following characterization of the periodic patterns of the signed shift $\Sigma_{\sigma}$, except in the case of the reverse shift. Throughout the paper we assume that $k \geq 2$.

Theorem 2.1 Let $\sigma \in\{+,-\}^{k}, \sigma \neq-{ }^{k}$. Then $\pi \in \mathcal{P}\left(\Sigma_{\sigma}\right)$ if and only if $\hat{\pi} \in \mathcal{C}^{\sigma}$.

This theorem, whose proof will require a few lemmas, states that the map $\theta$ gives a bijection between $\overline{\mathcal{P}}_{n}\left(\Sigma_{\sigma}\right)$ and $\mathcal{C}_{n}^{\sigma}$. The following lemma describes some conditions satisfied by the periodic patterns of $\Sigma_{\sigma}$, proving the forward direction of Theorem 2.1 .

Lemma 2.2 Let $\sigma \in\{+,-\}^{k}$, let $\pi \in \mathcal{P}_{n}\left(\Sigma_{\sigma}\right)$, and let $s=\left(s_{1} \ldots s_{n}\right)^{\infty} \in \mathcal{W}_{k}$ be such that $\pi=$ $\operatorname{PP}\left(s, \Sigma_{\sigma}\right)$. For $1 \leq t \leq k$, let $d_{t}=\left|\left\{i \in[n]: s_{i}<t\right\}\right|$, and let $d_{0}=0$. The following statements hold.

(a) For every $i$ and $t$, we have $d_{t}<\pi_{i} \leq d_{t+1}$ if and only if $s_{i}=t$.

(b) If $d_{t}<\pi_{i}<\pi_{j} \leq d_{t+1}$, then $\pi_{i+1}<\pi_{j+1}$ if $t \in T_{\sigma}^{+}$, and $\pi_{i+1}>\pi_{j+1}$ if $t \in T_{\sigma}^{-}$, where $\pi_{n+1}:=\pi_{1}$.

(c) For $0 \leq t<k, \hat{\pi}_{d_{t}+1} \ldots \hat{\pi}_{d_{t+1}}$ is increasing if $t \in T_{\sigma}^{+}$and decreasing if $t \in T_{\sigma}^{-}$. In particular, $\hat{\pi} \in \mathcal{C}^{\sigma}$.

Proof: Since $\operatorname{PP}\left(s, \Sigma_{\sigma}\right)=\pi$, it is clear for all $a, b \in[n], \pi_{a}<\pi_{b}$ implies $s_{a} \leq s_{b}$, from where (a) follows. To prove (b), suppose that $d_{t}<\pi_{i}<\pi_{j} \leq d_{t+1}$, and so $s_{[i, \infty)} \prec_{\sigma} s_{[j, \infty)}$. By part (a), we have $s_{i}=s_{j}=t$. If $t \in T_{\sigma}^{+}$, then $s_{[i+1, \infty)} \prec_{\sigma} s_{[j+1, \infty)}$, and so $\pi_{i+1}<\pi_{j+1}$. Similarly, if $t \in T_{\sigma}^{-}$, then $s_{[j+1, \infty)} \prec_{\sigma} s_{[i+1, \infty)}$, and so $\pi_{i+1}>\pi_{j+1}$.

Now let $0 \leq t<k$, and suppose that the indices $j$ such that $s_{j}=t$ are $j_{1}, \ldots, j_{m}$, ordered in such a way that $\pi_{j_{1}}<\pi_{j_{2}}<\cdots<\pi_{j_{m}}$, where $m=d_{t+1}-d_{t}$. Then part (a) implies that $\pi_{j_{\ell}}=d_{t-1}+\ell$ for $1 \leq \ell \leq$ $m$, and part (b) implies that $\pi_{j_{1}+1}<\pi_{j_{2}+1}<\cdots<\pi_{j_{m}+1}$ if $t \in T_{\sigma}^{+}$, and $\pi_{j_{1}+1}>\pi_{j_{2}+1}>\cdots>\pi_{j_{m}+1}$ if $t \in T_{\sigma}^{-}$. Using that $\pi_{j_{\ell}+1}=\hat{\pi}_{\pi_{j_{\ell}}}=\hat{\pi}_{d_{t}+\ell}$, this is equivalent to $\hat{\pi}_{d_{t}+1}<\hat{\pi}_{d_{t}+2}<\cdots<\hat{\pi}_{d_{t}+m}$ if $t \in T_{\sigma}^{+}$, and $\hat{\pi}_{d_{t}+1}>\hat{\pi}_{d_{t}+2}>\cdots>\hat{\pi}_{d_{t}+m}$ if $t \in T_{\sigma}^{-}$. Note that $d_{t}+m=d_{t+1}$. Since $\hat{\pi}$ is a cyclic permutation, this proves that $\hat{\pi} \in \mathcal{C}^{\sigma}$.

The next two lemmas will be used in the proof of the backward direction of Theorem 2.1 
Lemma 2.3 Let $\sigma \in\{+,-\}^{k}$, let $\pi \in \mathcal{S}_{n}$, and suppose that $\hat{\pi}=\hat{\pi}_{e_{0}+1} \ldots \hat{\pi}_{e_{1}} \hat{\pi}_{e_{1}+1} \ldots \hat{\pi}_{e_{2}} \ldots \hat{\pi}_{e_{k}}$, where each segment $\hat{\pi}_{e_{t}+1} \ldots \hat{\pi}_{e_{t+1}}$ is increasing if $t \in T_{\sigma}^{+}$and decreasing if $t \in T_{\sigma}^{-}$(and so $\hat{\pi} \in \mathcal{C}^{\sigma}$ ). Suppose that $e_{t}<\pi_{i}<\pi_{j} \leq e_{t+1}$ for some $1 \leq i, j \leq n$. Then $\pi_{i+1}<\pi_{j+1}$ ift $\in T_{\sigma}^{+}$, and $\pi_{i+1}>\pi_{j+1}$ if $t \in T_{\sigma}^{-}$, where $\pi_{n+1}:=\pi_{1}$.

Proof: Since $e_{t}<\pi_{i}<\pi_{j} \leq e_{t+1}$, both $\hat{\pi}_{\pi_{i}}$ and $\hat{\pi}_{\pi_{j}}$ lie in the segment $\hat{\pi}_{e_{t}+1} \ldots \hat{\pi}_{e_{t+1}}$. If $t \in T_{\sigma}^{+}$, this segment is increasing, so $\pi_{i+1}=\hat{\pi}_{\pi_{i}}<\hat{\pi}_{\pi_{j}}=\pi_{j+1}$. The argument is analogous if $t \in T_{\sigma}^{-}$.

Lemma 2.4 Let $\sigma \in\{+,-\}^{k}$ be arbitrary. If $\sigma=-{ }^{k}$, additionally assume that $n \neq 2 \bmod 4$. Let $\pi \in \mathcal{S}_{n}$ be such that $\hat{\pi} \in \mathcal{C}^{\sigma}$. Then there exist $0=e_{0} \leq e_{1} \leq \cdots \leq e_{k}=n$ such that

(a) each segment $\hat{\pi}_{e_{t}+1} \ldots \hat{\pi}_{e_{t+1}}$ is increasing if $t \in T_{\sigma}^{+}$and decreasing if $\in T_{\sigma}^{-}$; and

(b) the word $s_{1} \ldots s_{n}$, defined by $s_{i}=t$ whenever $e_{t}<\pi_{i} \leq e_{t+1}$, is primitive, and $s=\left(s_{1} \ldots s_{n}\right)^{\infty}$ satisfies $\mathrm{PP}\left(s, \Sigma_{\sigma}\right)=\pi$.

Furthermore, if $\sigma=+^{k}$ or $\sigma=-{ }^{k}$, then any choice of $0=e_{0} \leq e_{1} \leq \cdots \leq e_{k}=n$ satisfying (a) also satisfies (b).

Proof: Since $\hat{\pi} \in \mathcal{C}^{\sigma}$, there is some choice of $0=e_{0} \leq e_{1} \leq \cdots \leq e_{k}=n$ such that each segment $\hat{\pi}_{e_{t}+1} \ldots \hat{\pi}_{e_{t+1}}$ is increasing if $t \in T_{\sigma}^{+}$and decreasing if $t \in T_{\sigma}^{-}$. Pick one such choice and define $s_{1} \ldots s_{n}$ as above. In this proof we take the indices of $\pi \bmod n$, that is, we define $\pi_{i+j n}=\pi_{i}$ for $i \in[n]$.

Suppose that $s_{1} \ldots s_{n}$ is not primitive, so it can be written as $q^{m}$ for some $m \geq 2$ and some primitive word $q$ with $|q|=r=n / m$. Then, $s_{i}=s_{i+r}$ for all $i$. Let $g=\left|\left\{i \in[r]: s_{i} \in T_{\sigma}^{-}\right\}\right|$. Fix $i$, and let $t=s_{i}=s_{i+r}$. Because of the way that $s_{1} \ldots s_{n}$ is defined, we must have $e_{t}<\pi_{i}, \pi_{i+r} \leq e_{t+1}$, so we can apply Lemma 2.3 to this pair.

Suppose first that $g$ is even. If $\pi_{i}<\pi_{i+r}$, then applying Lemma $2.3 r$ times we get $\pi_{i+r}<\pi_{i+2 r}$, since the inequality involving $\pi_{i+\ell}$ and $\pi_{i+r+\ell}$ switches exactly $g$ times as $\ell$ increases from 0 to $r$. Starting with $i=1$ and applying this argument repeatedly, we see that if $\pi_{1}<\pi_{1+r}$, then $\pi_{1}<\pi_{1+r}<\pi_{1+2 r}<\cdots<$ $\pi_{1+(m-1) r}<\pi_{1+m r}=\pi_{1}$, which is a contradiction. A symmetric argument shows that if $\pi_{1}>\pi_{1+r}$, then $\pi_{1}>\pi_{1+r}>\pi_{1+2 r}>\cdots>\pi_{1+(m-1) r}>\pi_{1+m r}=\pi_{1}$.

It remains to consider the case that $g$ is odd. If $m$ is even and $m \geq 4$, then letting $q^{\prime}=q q$ we have $s_{1} s_{2} \ldots s_{n}=\left(q^{\prime}\right)^{\frac{m}{2}}$. Letting $r^{\prime}=\left|q^{\prime}\right|=2 r$ and $g^{\prime}=\left|\left\{i \in[2 r]: s_{i} \in T_{\sigma}^{-}\right\}\right|=2 g$, the same argument as above using $r^{\prime}$ and $g^{\prime}$ yields a contradiction. If $m$ is odd, suppose without loss of generality that $\pi_{1}<\pi_{1+r}$. Applying Lemma $2.3 r$ times to the inequality $\pi_{i}<\pi_{i+r}$ (respectively $\pi_{i}>\pi_{i+r}$ ) yields $\pi_{i+r}>\pi_{i+2 r}$ (respectively $\pi_{i+r}<\pi_{i+2 r}$ ) in this case, since the inequality involving $\pi_{i+\ell}$ and $\pi_{i+r+\ell}$ switches an odd number of times. Consider two cases:

- If $\pi_{1}<\pi_{1+2 r}$, then Lemma 2.3 applied repeatedly in blocks of $2 r$ times yields $\pi_{1}<\pi_{1+2 r}<$ $\pi_{1+4 r}<\cdots<\pi_{1+(m-1) r}$. Applying now Lemma $2.3 r$ times starting with $\pi_{1}<\pi_{1+(m-1) r}$ gives $\pi_{1+r}>\pi_{1+m r}=\pi_{1}$, which contradicts the assumption $\pi_{1}<\pi_{1+r}$.

- If $\pi_{1}>\pi_{1+2 r}$, applying Lemma $2.3 r$ times we get $\pi_{1+r}<\pi_{1+3 r}$, and by repeated application of the lemma in blocks of $2 r$ times it follows that $\pi_{1+r}<\pi_{1+3 r}<\pi_{1+5 r}<\cdots<\pi_{1+(m-2) r}<$ $\pi_{1+m r}=\pi_{1}$, contradicting again the assumption $\pi_{1}<\pi_{1+r}$. 
The only case left is when $g$ is odd and $m=2$, that is, when $s_{1} s_{2} \ldots s_{n}=q^{2}$ and $q$ has an odd number of letters in $T_{\sigma}^{-}$. Note that this situation does not happen when $\sigma=+^{k}$ (since in this case $g=0$ ) and, although it can happen when $\sigma=-^{k}$, in this case we would have that $T_{\sigma}^{-}=\{0,1, \ldots, k-1\}$, and so $n=2 r=2 g=2 \bmod 4$, which we are excluding in the statement of the theorem.

Thus, we can assume that there exists some $1 \leq \ell<k$ such that $\sigma_{\ell-1} \sigma_{\ell}$ is either +- or -+ . We will show that there is a choice of $0=e_{0}^{\prime} \leq e_{1}^{\prime} \leq \cdots \leq e_{k}^{\prime}=n$ that satisfies the conditions of the lemma, and the resulting word $s_{1}^{\prime} s_{2}^{\prime} \ldots s_{n}^{\prime}$ is primitive.

Suppose that $\sigma_{\ell-1} \sigma_{\ell}=+-$ (the case $\sigma_{\ell-1} \sigma_{\ell}=-+$ is very similar). Then, $\hat{\pi}_{e_{\ell-1}+1}<\cdots<\hat{\pi}_{e_{\ell}}$ and $\hat{\pi}_{e_{\ell}+1}>\cdots>\hat{\pi}_{e_{\ell+1}}$. If $\hat{\pi}_{e_{\ell}}<\hat{\pi}_{e_{\ell}+1}$ (respectively, $\hat{\pi}_{e_{\ell}}>\hat{\pi}_{e_{\ell}+1}$ ), let $e_{\ell}^{\prime}:=e_{\ell}+1$ (respectively, $e_{\ell}^{\prime}:=e_{\ell}-1$ ), and $e_{t}^{\prime}:=e_{t}$ for all $t \neq \ell$. Clearly, the values $e_{t}^{\prime}$ satisfy part (a) of the lemma. Additionally, the word $s_{1}^{\prime} \ldots s_{n}^{\prime}$ that they define using part (b) differs from the original word $s_{1} s_{2} \ldots s_{n}=q^{2}$ by one entry, making the number of $\ell$ s that appear in $s_{1}^{\prime} \ldots s_{n}^{\prime}$ be odd instead of even. Thus, $s_{1}^{\prime} \ldots s_{n}^{\prime}$ can no longer be written as $\left(q^{\prime}\right)^{2}$ for any $q^{\prime}$, so it is primitive by the above argument.

Finally, we prove that if we let $s=\left(s_{1} \ldots s_{n}\right)^{\infty}$, then $\mathrm{PP}\left(s, \Sigma_{\sigma}\right)=\pi$. Let $1 \leq i, j \leq n$ with $\pi_{i}<\pi_{j}$. We need to show that $s_{[i, \infty)} \prec_{\sigma} s_{[j, \infty)}$. Let $a \geq 0$ be the smallest such that $s_{i+a} \neq s_{j+a}$, and let $h=\left|\left\{0 \leq \ell \leq a-1: s_{\ell} \in T_{\sigma}^{-}\right\}\right|$. If $h$ is even, then Lemma2.3 applied $a$ times shows that $\pi_{i+a}<\pi_{j+a}$. Since $s_{i+a} \neq s_{j+a}$, we must then have $s_{i+a}<s_{j+a}$, by construction of $s$. Thus, $s_{[i, \infty)} \prec_{\sigma} s_{[j, \infty)}$ by definition of $\prec_{\sigma}$, since the word $s_{i} s_{i+1} \ldots s_{i+a-1}=s_{j} s_{j+1} \ldots s_{j+a-1}$ has an even number of letters in $T_{\sigma}^{-}$. Similarly, if $h$ is odd, then Lemma 2.3 shows that $\pi_{i+a}>\pi_{j+a}$. Since $s_{i+a} \neq s_{j+a}$, we must have $s_{i+a}>s_{j+a}$, and thus $s_{[i, \infty)} \prec_{\sigma} s_{[j, \infty)}$ by definition of $\prec_{\sigma}$.

We can now combine the above lemmas to prove our main theorem.

Proof of Theorem 2.1: If $\pi \in \mathcal{P}\left(\Sigma_{\sigma}\right)$, then $\hat{\pi} \in \mathcal{C}^{\sigma}$ by Lemma 2.2 (c). Conversely, $\pi \in \mathcal{S}_{n}$ is such that $\hat{\pi} \in \mathcal{C}^{\sigma}$, then the word $s$ given by Lemma 2.4 (b) satisfies $\operatorname{PP}\left(s, \Sigma_{\sigma}\right)=\pi$, and so $\pi \in \mathcal{P}\left(\Sigma_{\sigma}\right)$.

For $\sigma={ }^{k}$, the same proof yields the following weaker result.

Proposition 2.5 Let $\sigma=-{ }^{k}$. If $\pi \in \mathcal{P}_{n}\left(\Sigma_{\sigma}\right)$, then $\hat{\pi} \in \mathcal{C}_{n}^{\sigma}$. Additionally, the converse holds if $n \neq$ $2 \bmod 4$.

Define the reversal of $\sigma=\sigma_{0} \sigma_{1} \ldots \sigma_{k-1}$ to be $\sigma^{R}=\sigma_{k-1} \ldots \sigma_{1} \sigma_{0}$. If $\pi \in \mathcal{S}_{n}$, then the complement of $\pi$ is the permutation $\pi^{c}$ where $\pi_{i}^{c}=n+1-\pi_{i}$ for $1 \leq i \leq n$. The following result, whose proof is omitted, relates periodic patterns of $\Sigma_{\sigma}$ and $\Sigma_{\sigma R}$.

Proposition 2.6 For every $\sigma \in\{+,-\}^{k}, \pi \in \mathcal{P}\left(\Sigma_{\sigma}\right)$ if and only if $\pi^{c} \in \mathcal{P}\left(\Sigma_{\sigma^{R}}\right)$.

\section{Enumeration for special cases}

For particular values of $\sigma$, we can give a formula for the number of periodic patterns of $\Sigma_{\sigma}$. This is the case when $\sigma=+-, \sigma=+{ }^{k}$, and $\sigma=-{ }^{k}$, for any $k \geq 2$.

\subsection{The tent map}

We denote the tent map by $\Lambda=\Sigma_{+-}$. The characterization of the periodic patterns of $\Lambda$ follows from Theorem 2.1

Corollary 3.1 $\pi \in \mathcal{P}(\Lambda)$ if and only if $\hat{\pi}$ is unimodal. 
Next we give an exact formula for the number of periodic patterns of the tent map.

Theorem 3.2

$$
p_{n}(\Lambda)=\frac{1}{2 n} \sum_{\substack{d \mid n \\ d \text { odd }}} \mu(d) 2^{\frac{n}{d}} .
$$

Proof: Let $\mathcal{O}_{n}$ be the set of binary words $s=\left(s_{1} \ldots s_{n}\right)^{\infty}$ where $s_{1} \ldots s_{n}$ is primitive and has an odd number of ones. We will show that the map $s \mapsto \operatorname{PP}(s, \Lambda)$ is a bijection between $\mathcal{O}_{n}$ and $\mathcal{P}_{n}(\Lambda)$. It is clear that this map is well defined. We will prove that for each $\pi \in \mathcal{P}_{n}(\Lambda)$ there are either one or two periodic binary words $s$ such that $\operatorname{PP}(s, \Lambda)=\pi$, and that exactly one of them is in $\mathcal{O}_{n}$.

Fix $\pi \in \mathcal{P}_{n}(\Lambda)$, and recall from Corollary 3.1 that $\hat{\pi}_{1}<\hat{\pi}_{2}<\cdots<\hat{\pi}_{m}>\hat{\pi}_{m+1}>\cdots>\hat{\pi}_{n}$ for some $m$. Let $s=\left(s_{1} \ldots s_{n}\right)^{\infty}$ be such that $\mathrm{PP}(s, \Lambda)=\pi$, and let $d=\left|\left\{1 \leq i \leq n: s_{i}=0\right\}\right|$. By Lemma 2.2 a), we have that $s_{i}=0$ if and only if $\pi_{i} \leq d$. Suppose now that $s_{i}=s_{j}$ and $\pi_{i}<\pi_{j}$. If $s_{i}=s_{j}=0$, then $\pi_{i+1}<\pi_{j+1}$ by Lemma 2.2 b), and so $\hat{\pi}_{\pi_{i}}<\hat{\pi}_{\pi_{j}}$. Since this holds whenever $1 \leq \pi_{i}<\pi_{j} \leq d$, we see that $\hat{\pi}_{1}<\hat{\pi}_{2}<\cdots<\hat{\pi}_{d}$. Similarly, if $s_{i}=s_{j}=1$, then $\pi_{i+1}>\pi_{j+1}$ and so $\hat{\pi}_{\pi_{i}}>\hat{\pi}_{\pi_{j}}$. Thus, $\hat{\pi}_{d+1}>\hat{\pi}_{d+2}>\cdots>\hat{\pi}_{n}$.

It follows that $m=d$ or $m=d+1$, depending on whether $\hat{\pi}_{d}>\hat{\pi}_{d+1}$ or $\hat{\pi}_{d}<\hat{\pi}_{d+1}$. Thus, since $\pi$ was fixed, there are two choices for $d$, namely $d=m$ or $d=m-1$. This corresponds to setting $s_{i}$, where $i$ is such that $\pi_{i}=m$, equal to 1 or to 0 , respectively. The above argument shows that the rest of the entries of $s$ are forced by $\pi$. For exactly one of these two choices, $s_{1} \ldots s_{n}$ will have an odd number of ones.

However, as shown in the proof of Theorem 2.1 it is possible for $s_{1} \ldots s_{n}$ constructed as above not to be primitive. This can only happen when $n$ is even and $s_{1} \ldots s_{n}=q^{2}$, in which case $s_{1} \ldots s_{n}$ has an even number of ones. Thus, the choice where $s_{1} \ldots s_{n}$ has an odd number of ones is primitive, so $s \in \mathcal{O}_{n}$ and it satisfies $\operatorname{PP}(s, \Lambda)=\pi$.

Using the Möbius inversion formula, it can be shown that the number of primitive binary words of length $n$ with an odd number of ones is $\left|\mathcal{O}_{n}\right|=\sum_{d} \mu(d) 2^{n / d-1}$, where the sum is over all odd divisors of $n$. Since $p_{n}(\Lambda)=\left|\mathcal{O}_{n}\right| / n$, the formula follows.

\subsection{The $k$-shift}

Recall that the $k$-shift is the map $\Sigma_{\sigma}$ where $\sigma=+{ }^{k}$. We denote this map by $\Sigma_{k}$ for convenience. The allowed patterns of the $k$-shift were characterized and enumerated by Elizalde [8], building up on work by Amigó et al. [3].

In this section we describe and enumerate the periodic patterns of the $k$-shift. Denote the descent set of $\pi \in \mathcal{S}_{n}$ by $\operatorname{Des}(\pi)=\left\{i \in[n-1]: \pi_{i}>\pi_{i+1}\right\}$, and by $\operatorname{des}(\pi)=|\operatorname{Des}(\pi)|$ the number of descents of $\pi$. In the case of the $k$-shift, Theorem 2.1 states that $\pi \in \mathcal{P}\left(\Sigma_{k}\right)$ if and only if $\hat{\pi}$ is a cyclic permutation that can be written as a concatenation of $k$ increasing sequences. The following corollary follows from this description.

Corollary $3.3 \pi \in \mathcal{P}\left(\Sigma_{k}\right)$ if and only if $\operatorname{des}(\hat{\pi}) \leq k-1$.

An equivalent statement is that $\theta$ gives a bijection between $\overline{\mathcal{P}}_{n}\left(\Sigma_{k}\right)$ and permutations in $\mathcal{C}_{n}$ with at most $k-1$ descents. It will be convenient to define, for $1 \leq i \leq n$,

$$
C(n, i)=\left|\left\{\tau \in \mathcal{C}_{n}: \operatorname{des}(\tau)=i-1\right\}\right| .
$$


In the rest of this section we assume that $n \geq 2$. We start by giving a formula for the number of periodic patterns of the binary shift. Recall the formula for $L_{k}(n)$ given in Lemma 1.1 .

Theorem 3.4 For $n \geq 2$, we have $p_{n}\left(\Sigma_{2}\right)=C(n, 2)=L_{2}(n)$.

Proof: When $n \geq 2$, there are no permutations in $\mathcal{C}_{n}$ with no descents, so Corollary 3.3 states that $\pi \in \mathcal{P}\left(\Sigma_{2}\right)$ if and only if $\operatorname{des}(\hat{\pi})=1$. It follows that $\overline{\mathcal{P}}_{n}\left(\Sigma_{2}\right)$ is in bijection with permutations in $\mathcal{C}_{n}$ with exactly one descent, so $p_{n}\left(\Sigma_{2}\right)=C(n, 2)$.

Next we show that $\overline{\mathcal{P}}_{n}\left(\Sigma_{2}\right)$ is also in bijection with the set of periodic orbits of size $n$ of $\Sigma_{2}$, and thus $p_{n}\left(\Sigma_{2}\right)=L_{2}(n)$ by Lemma 1.1 Clearly, to each $n$-periodic point $s=\left(s_{1} \ldots s_{n}\right)^{\infty}$ one can associate the periodic pattern $\pi=\operatorname{PP}\left(s, \Sigma_{2}\right) \in \mathcal{P}_{n}\left(\Sigma_{2}\right)$, so that the $n$ points in the orbit of $s$ give rise to the patterns in $[\pi]$. Conversely, for each $\pi \in \mathcal{P}_{n}\left(\Sigma_{2}\right)$ there is some $s \in \mathcal{W}_{2}$ such that $\operatorname{PP}\left(s, \Sigma_{2}\right)=\pi$. It remains to show that $s$ is unique. Suppose that $\operatorname{Des}(\hat{\pi})=\{j\}$ and that $\operatorname{PP}\left(s, \Sigma_{2}\right)=\pi$. Letting $d$ be the number of zeros in $s_{1} \ldots s_{n}$, we have by Lemma 2.2 (c) that $\hat{\pi}_{1}<\cdots<\hat{\pi}_{d}$ and $\hat{\pi}_{d+1}<\cdots<\hat{\pi}_{n}$. Thus, $d=j$, and so the word $s_{1} \ldots s_{n}$ is uniquely determined by Lemma 2.2 (a).

Theorem 3.5 For $k \geq 3$ and $n \geq 2$,

$$
p_{n}\left(\Sigma_{k}\right)-p_{n}\left(\Sigma_{k-1}\right)=C(n, k)=L_{k}(n)-\sum_{i=2}^{k-1}\left(\begin{array}{c}
n+k-i \\
k-i
\end{array}\right) C(n, i) .
$$

Proof: It is clear from Corollary 3.3 that $C(n, k)=\frac{1}{n}\left|\mathcal{P}_{n}\left(\Sigma_{k}\right) \backslash \mathcal{P}_{n}\left(\Sigma_{k-1}\right)\right|=p_{n}\left(\Sigma_{k}\right)-p_{n}\left(\Sigma_{k-1}\right)$.

To prove the recursive formula for $C(n, k)$, we count periodic orbits of size $n$ of $\Sigma_{k}$ in two ways. On one hand, this number equals $L_{k}(n)$ by Lemma 1.1. On the other hand, to each such orbit one can associate an equivalence class $[\pi] \in \overline{\mathcal{P}}_{n}\left(\Sigma_{k}\right)$, consisting of the periodic patterns at the $n$ points of the orbit.

Fix $\pi \in \mathcal{P}_{n}\left(\Sigma_{k}\right)$. We now count how many words $s \in \mathcal{W}_{k}$ satisfy $\operatorname{PP}\left(s, \Sigma_{k}\right)=\pi$ (equivalently, how many periodic orbits are associated with $[\pi]$ ). By Lemma 2.4 for each choice of $0=e_{0} \leq e_{1} \leq \cdots \leq$ $e_{k}=n$ such that $\hat{\pi}_{e_{t}+1} \ldots \hat{\pi}_{e_{t+1}}$ is increasing for all $0 \leq t<k$, the word $s$ defined in part (b) of the lemma satisfies $\operatorname{PP}\left(s, \Sigma_{k}\right)=\pi$. Conversely, if $s=\left(s_{1} \ldots s_{n}\right)^{\infty} \in \mathcal{W}_{k}$ is such that $\operatorname{PP}\left(s, \Sigma_{k}\right)=\pi$, then, by Lemma 2.2(c), each block $\hat{\pi}_{d_{t}+1} \ldots \hat{\pi}_{d_{t+1}}$ is increasing, with $d_{t}$ defined as in the lemma, for $0 \leq t<k$. Thus, finding all the words $s \in \mathcal{W}_{k}$ such that $\operatorname{PP}\left(s, \Sigma_{k}\right)=\pi$ is equivalent to finding all the ways to choose $0=e_{0} \leq e_{1} \leq \cdots \leq e_{k}=n$ such that $\hat{\pi}_{e_{t}+1} \ldots \hat{\pi}_{e_{t+1}}$ is increasing for all $0 \leq t<k$. If $\operatorname{des}(\hat{\pi})=i-1$, it is a simple exercise to show that there are $\left(\begin{array}{c}n+k-i \\ k-i\end{array}\right)$ such choices, since $\operatorname{Des}(\hat{\pi})$ has to be a subset of $\left\{e_{1}, \ldots, e_{k-1}\right\}$.

By Corollary 3.3 . for each $2 \leq i \leq k$, the number of equivalence classes $[\pi] \in \overline{\mathcal{P}}_{n}\left(\Sigma_{k}\right)$ where $\operatorname{des}(\hat{\pi})=i-1$ is $C(n, i)$. It follows that

$$
L_{k}(n)=\sum_{i=2}^{k}\left(\begin{array}{c}
n+k-i \\
k-i
\end{array}\right) C(n, i),
$$

which is equivalent to the stated formula.

It follows immediately from Theorem 3.5 that $p_{n}\left(\Sigma_{k}\right)=\sum_{i=2}^{k} C(n, i)$ for $n \geq 2$. 
Let us show an example that illustrates how, in the above proof, the words $s \in \mathcal{W}_{k}$ with $\operatorname{PP}\left(s, \Sigma_{k}\right)=\pi$ are constructed for given $\pi$. Let $k=5$, and let $\pi=165398427 \in \mathcal{P}_{9}\left(\Sigma_{5}\right)$. Then $\hat{\pi}=679235148$, which has descent set $\operatorname{Des}(\hat{\pi})=\{3,6\}$. Choosing $e_{1}=3, e_{2}=6, e_{3}=e_{4}=9$, Lemma 2.4 gives the word $s_{1} \ldots s_{9}=011022102$. Choosing $e_{1}=2, e_{2}=3, e_{3}=6, e_{4}=7$, we get $s_{1} \ldots s_{9}=022144203$.

The second equality in Theorem 3.5]also follows from a result of Gessel and Reutenauer [11, Theorem 6.1 , which is proved using quasi-symmetric functions.

\subsection{The reverse $k$-shift, when $n \neq 2 \bmod 4$}

The reverse $k$-shift is the map $\Sigma_{\sigma}$ where $\sigma=-{ }^{k}$. We denote this map by $\Sigma_{k}^{-}$in this section. Denote the ascent set of $\pi \in \mathcal{S}_{n}$ by $\operatorname{Asc}(\pi)=\left\{i \in[n-1]: \pi_{i}<\pi_{i+1}\right\}$, and the number of ascents of $\pi$ by $\operatorname{asc}(\pi)=|\operatorname{Asc}(\pi)|=n-1-\operatorname{des}(\pi)$.

Proposition 2.5 gives a partial characterization of the periodic patterns of $\Sigma_{k}^{-}$. For patterns of length $n \neq 2 \bmod 4$, it states that $\pi \in \mathcal{P}_{n}\left(\Sigma_{k}^{-}\right)$if and only if $\hat{\pi}$ can be written as a concatenation of $k$ decreasing sequences. The next corollary follows from this description. The case $n=2 \bmod 4$ will be discussed in Section 3.4 .

Corollary 3.6 Let $\pi \in \mathcal{S}_{n}$, where $n \neq 2 \bmod 4$. Then $\pi \in \mathcal{P}\left(\Sigma_{k}^{-}\right)$if and only if $\operatorname{asc}(\hat{\pi}) \leq k-1$.

To enumerate periodic patterns of $\Sigma_{k}^{-}$of length $n \neq 2 \bmod 4$, we use an argument very similar to the one we used for $\Sigma_{k}$. For $1 \leq i \leq n$, let $C^{\prime}(n, i)=\left|\left\{\tau \in \mathcal{C}_{n}: \operatorname{asc}(\tau)=i-1\right\}\right|$. By definition, we have $C^{\prime}(n, i)=C(n, n-i+1)$. The proofs of the following two theorems are similar to those of Theorems 3.4 and 3.5 and thus omitted from this extended abstract.

Theorem 3.7 For $n \geq 3$ with $n \neq 2 \bmod 4$, we have $p_{n}\left(\Sigma_{2}^{-}\right)=C^{\prime}(n, 2)=L_{2}(n)$.

Theorem 3.8 For $n \geq 3$ with $n \neq 2 \bmod 4$ and $k \geq 3$,

$$
p_{n}\left(\Sigma_{k}\right)-p_{n}\left(\Sigma_{k-1}\right)=C^{\prime}(n, k)=L_{k}(n)-\sum_{i=2}^{k-1}\left(\begin{array}{c}
n+k-i \\
k-i
\end{array}\right) C^{\prime}(n, i) .
$$

Combining Theorems 3.4, 3.5, 3.7 and 3.8, we obtain the following.

Corollary 3.9 For $n \neq 2 \bmod 4$ and $2 \leq k \leq n$, we have $C(n, k)=C^{\prime}(n, k)$.

This equality is equivalent to the symmetry $C(n, k)=C(n, n-1-k)$, which is not obvious from the recursive formula in Theorem 3.5. Corollary 3.9 also follows from a more general result of Gessel and Reutenauer [11, Theorem 4.1], which states that if $n \neq 2 \bmod 4$, then for any $D \subseteq[n-1]$,

$$
\left|\left\{\tau \in \mathcal{C}_{n}: \operatorname{Des}(\tau)=D\right\}\right|=\left|\left\{\tau \in \mathcal{C}_{n}: \operatorname{Asc}(\tau)=D\right\}\right|
$$

Their proof involves quasi-symmetric functions. Even though describing a direct bijection proving Eq. (1) remains an open problem, our construction can be used to give the following bijection between $\left\{\tau \in \mathcal{C}_{n}\right.$ : $\operatorname{Des}(\tau) \subseteq D\}$ and $\left\{\tau \in \mathcal{C}_{n}: \operatorname{Asc}(\tau) \subseteq D\right\}$

Given $\hat{\pi} \in \mathcal{C}_{n}$ such that $\operatorname{Des}(\hat{\pi}) \subseteq D=\left\{d_{1}, d_{2}, \ldots, d_{k-1}\right\}$, let $\pi \in \mathcal{S}_{n}$ be such that $\theta(\pi)=\hat{\pi}$ and $\pi_{1}=1$. Let $s=\left(s_{1} \ldots s_{n}\right)^{\infty} \in \mathcal{W}_{k}$ be defined by $s_{i}=t$ if $d_{t}<\pi_{i} \leq d_{t+1}$, for $1 \leq i \leq n$, where we let $d_{0}=0$ and $d_{k}=n$. Let $\pi^{\prime}=\operatorname{PP}\left(s, \Sigma_{k}^{-}\right)$. Then $\hat{\pi}^{\prime}=\theta\left(\pi^{\prime}\right) \in \mathcal{C}_{n}, \operatorname{Asc}\left(\hat{\pi^{\prime}}\right) \subseteq D$, and the map $\hat{\pi} \mapsto \hat{\pi^{\prime}}$ gives the desired bijection.

Another consequence of Theorem 3.8 is that $p_{n}\left(\Sigma_{k}^{-}\right)=\sum_{i=2}^{k} C^{\prime}(n, k)=p_{n}\left(\Sigma_{k}\right)$ when $n \neq 2 \bmod 4$. 


\subsection{The reverse $k$-shift, when $n=2$ mod 4}

When $n=2 \bmod 4$, the results in Section 3.3 no longer hold. Corollary 3.6 fails in that there are certain permutations $\pi \in \mathcal{S}_{n}$ with asc $(\hat{\pi}) \leq k-1$ that are not periodic patterns for $\Sigma_{k}^{-}$. For the binary case, the number of periodic patterns of $\Sigma_{2}^{-}$is given next.

Theorem 3.10 For $n \geq 3$ with $n=2 \bmod 4$,

$$
p_{n}\left(\Sigma_{2}^{-}\right)=L_{2}(n)=C^{\prime}(n, 2)-C^{\prime}(n / 2,2) .
$$

The proof of this theorem, which we omit due to lack of space, is based in the following idea. By Proposition 2.5, the map $\theta$ gives an injection from $\overline{\mathcal{P}}_{n}\left(\Sigma_{2}^{-}\right)$to the set $\left\{\tau \in \mathcal{C}_{n}: \operatorname{asc}(\tau)=1\right\}$. To show that the number of cycles with one ascent that are not in the image of this map is precisely $C^{\prime}(n / 2,2)$, we give a bijection with primitive binary necklaces of length $r$, constructed by analyzing how Lemma 2.4 fails when $n=2 \bmod 4$. From Theorems 3.4, 3.7 and 3.10, we get the following.

\section{Corollary 3.11}

$$
C^{\prime}(n, 2)= \begin{cases}C(n, 2)+C(n / 2,2) & n=2 \bmod 4 \\ C(n, 2) & n \neq 2 \bmod 4\end{cases}
$$

For $k \geq 3$, the number of periodic patterns is given in the next theorem, whose proof is omitted.

Theorem 3.12 For $n \geq 3$ with $n=2 \bmod 4$ and $k \geq 3$,

$$
p_{n}\left(\Sigma_{k}^{-}\right)=\sum_{i=2}^{k} C^{\prime}(n, i)-C^{\prime}(n / 2, k) .
$$

Since $n / 2$ is odd in this case, $C^{\prime}(n / 2, k)$ is easily computed by the recurrence in Theorem 3.8 To compute $C^{\prime}(n, i)$, one can use the following recurrence.

Theorem 3.13 For $n \geq 3$ with $n=2 \bmod 4$ and $k \geq 3$,

$$
C^{\prime}(n, k)=L_{k}(n)-\sum_{i=2}^{k-1}\left[\left(\begin{array}{c}
n+k-i \\
k-i
\end{array}\right) C^{\prime}(n, i)-\left(\begin{array}{c}
n / 2+k-i \\
k-i
\end{array}\right) C^{\prime}(n / 2, i)\right]+C^{\prime}(n / 2, k) .
$$

\section{Pattern-avoiding cyclic permutations}

The enumeration of pattern-avoiding cycles is a wide-open problem, part of its difficulty stemming from the fact that it combines two different ways to look at permutations: in terms of their cycle structure and in terms of their one-line notation. The question of finding a formula for $\left|\mathcal{C}_{n}(\sigma)\right|$ where $\sigma$ is a pattern of length 3 was proposed by Richard Stanley and is still open. However, using Theorem 2.1 the formulas that we have found for the number of periodic patterns of the tent map, the $k$-shift and the reverse $k$-shift translate into the following related results.

Theorem 4.1 For $n \geq 2$,

$$
\left|\mathcal{C}_{n}(213,312)\right|=\frac{1}{2 n} \sum_{\substack{d \mid n \\ d \text { odd }}} \mu(d) 2^{n / d}, \quad\left|\mathcal{C}_{n}(321,2143,3142)\right|=\frac{1}{n} \sum_{d \mid n} \mu(d) 2^{n / d},
$$




$$
\left|\mathcal{C}_{n}(123,2413,3412)\right|= \begin{cases}\frac{1}{n} \sum_{d \mid n} \mu(d) 2^{n / d} & \text { if } n \neq 2 \bmod 4, \\ \frac{1}{n} \sum_{d \mid n} \mu(d) 2^{n / d}+\frac{2}{n} \sum_{d \mid \frac{n}{2}} \mu(d) 2^{n / 2 d} & \text { if } n=2 \bmod 4 .\end{cases}
$$

Proof: The formula for $\left|\mathcal{C}_{n}(213,312)\right|$ is a consequence of Theorem 3.2 and Corollary 3.1 , together with the fact that a permutation is unimodal if and only if it avoids 213 and 312 . The second formula follows from Theorem 3.4 using that the set of permutations with at most one descent is $\mathcal{S}^{++}=$ $\mathcal{S}(321,2143,3142)$ (see [5]). Finally, the third formula is a consequence of Corollary 3.11 and Theorem 3.4. noting that the class of permutations with at most one ascent is $\mathcal{S}(123,2413,3412)$.

\section{References}

[1] J.M. Amigó, The ordinal structure of the signed shift transformations, Internat. J. Bifur. Chaos Appl. Sci. Engrg. 19 (2009), 3311-3327.

[2] J.M. Amigó, Permutation complexity in dynamical systems, Springer Series in Synergetics, SpringerVerlag, Berlin, 2010.

[3] J.M. Amigó, S. Elizalde and M. Kennel, Forbidden patterns and shift systems, J. Combin. Theory Ser. A 115 (2008), 485-504.

[4] J.M. Amigó, S. Zambrano and M.A.F. Sanjuán, Detecting determinism in time series with ordinal patterns: a comparative study, Internat. J. Bifur. Chaos Appl. Sci. Engrg. 20 (2010), 2915-2924.

[5] M. Atkinson, Restricted permutations, Discrete Math. 195 (1999), 27-38.

[6] C. Bandt, G. Keller and B. Pompe, Entropy of interval maps via permutations, Nonlinearity 15 (2002), 1595-1602.

[7] C. Bernhardt, E. Coven, M. Misiurewicz and I. Mulvey, Comparing Periodic Orbits of Maps of the Interval, Trans. Amer. Math. Soc. 333 (1992), 701-707.

[8] S. Elizalde, The number of permutations realized by a shift, SIAM J. Discrete Math. 23 (2009), 765786.

[9] S. Elizalde, Permutations and $\beta$-shifts, J. Combin. Theory Ser. A 118 (2011), 2474-2497.

[10] S. Elizalde and Y. Liu, On basic forbidden patterns of functions, Discrete Appl. Math. 159 (2011), $1207-1216$.

[11] I. Gessel and C. Reutenauer, Counting Permutations with Given Cycle Structure and Descent Set. Journal of Combinatorial Theory. Series A 64, (1993) 189-215.

[12] I. Jungreis, Some Results on the Sarkovskii Partial Ordering of Permutations, Trans. Amer. Math. Soc. 325 (1991), 319-344.

[13] A.N. Sarkovskii, Coexistence of cycles of a continuous map of a line into itself, Ukrainian Math. J. 16 (1964), pp. 61-71. 